\title{
Bias-Correction In Localization Algorithms
}

\author{
Yiming Ji, Changbin Yu, Brian D.O. Anderson
}

\begin{abstract}
In this paper we introduce a new approach to determine the bias in localization algorithms by mixing Taylor series and Jacobian matrices, which results in an easily calculated analytical expression for the bias. To illustrate this approach, we analyze the proposed method in two situations using localization algorithms based on distance measurements. Monte Carlo simulations verify that the proposed method is consistent with the performance of localization algorithms, which means the bias-correction method can correct the bias in most situations except when there is a collinearity problem. Although the method is analyzed in distance-based localization algorithms, it can be extended to other kinds of localization algorithms.
\end{abstract}

Keywords: Localization; Bias correction; Sensor network.

\section{INTRODUCTION}

Accurate location of targets is required in many different application areas. For example in wireless sensor networks applications, such as bush fire surveillance, water quality monitoring and precision agriculture, thousands of sensors may have to be localized accurately. Again, in radar detection, the target position should be obtained precisely. In most situations, the measurement information is limited and inaccurate. Therefore, techniques for determining location of a target using restricted and noisy measurements have been widely investigated with many localization algorithms proposed in recent years, see e.g. [1-2].

However almost all current localization algorithms are affected by measurement errors, which means a true position cannot be obtained with noisy measurements. Once errors exist in measurements, the position estimates in localization will usually be biased. Nevertheless rather few works address the bias problem. Two kinds of bias have in fact been mentioned in localization problems. The first one is measurement bias, which means that bias-a systematic error in one directionexists in the measurement set. This is caused by the environmental conditions. In [3] J.S. Picard et al. discussed several models for bias in the range measurements, and presented a set of iterative algorithms that cope successfully with the various bias models and provide maximum likelihood position estimates. Again, X. Lin et al. [4] present a model of measurement bias in polar coordinates with an exact solution for the measurement bias estimation also provided in the paper.

The other type of bias is in estimation, which is the subject of this paper. In such a case, the measurements themselves, though perturbed by noise, are not biased. But the localization estimates are obtained through nonlinear processing of the measurements, and this gives rise to bias. Gavish et al. [5] present the analytical expressions of bias which permit performance comparison for two well known bearing only location techniques, viz. the maximum likelihood and the Stansfield estimators. To obtain the analytical expressions of bias, they expand the first derivative of the cost function to

Y. Ji and C. Yu are with Research School of Information Sciences and Engineering, the Australian National University Canberra ACT 2600, Australia. Email: \{yiming.ji, brad.yu\}@anu.edu.au

B.D.O. Anderson is with the Australian National University and National ICT Australia Ltd. Email: brian.anderson@anu.edu.au first-order and second-order separately. They obtain an analytical expression for the bias which includes the derivatives of the cost function and the variance of the measurement noise. However to calculate the bias directly in this way is complex. In [6], Doğançay et al. propose bias reduction techniques for the scan-based least-squares emitter localization algorithm. A weighted least-squares estimator and an iterative maximum likelihood estimator are developed to overcome the estimation bias. Nevertheless these bias reduction methods only aim at least-squares and maximum likelihood localization algorithms and thus are not generic. Drake and Doğançay [7] give a short introduction to tensor algebra and provide a few examples of how to use it. In the paper bias in non-linear systems with noisy input is discussed as one of the applications of tensor algebra. They expand the non-linear function, which maps measurements to target positions, to the second order in the noise by a Taylor series. The expected value of the second order term is considered as the bias. A simple example, which considers a radar measuring the location of a target in polar coordinates while reporting it in cartesian coordinates, is also considered in the paper.

In this paper, a general approach is presented to correct the bias in 2D localization algorithms. We first expand the localization mapping $g$ (which maps from the measurements to produce position estimates) by a Taylor series to second-order in the measurement noise, and we consider the expected values of the second-order term, which is computed by the derivatives of $g$, as bias. However, it is often very hard to calculate the derivatives of $g$ analytically. In contrast, the inverse mapping of $g$ (call it $f$ ) and its derivatives can be obtained much more easily. Therefore, we introduce the Jacobian matrix of $f$ to compute the derivatives of the localization mapping $g$ in terms of the derivatives of $f$, resulting in a simple calculation of bias. Our method is then applied by way of example to distancebased localization algorithms, though it is independent of the particular type of measurement or localization algorithm, i.e. the bias-correction method can be extended to other kinds of localization algorithms

The rest of the paper is organized as follows. In Section II high level views of localization and bias are summarized. We analyze the proposed method in two situations in Section III. The results of Monte Carlo simulations are provided in Section IV. Section V summarizes the paper and comments on future work.

\section{Problem Statement}

\section{A. High Level View of Localization}

Localization refers to the process of estimating the locations of target positions using the measurements. For example in wireless sensor networks, the position of an unlocalized node can be estimated by gathering the distance or bearing information from neighboring nodes whose position has already been identified. In this subsection a brief description of localization will be presented.

Suppose there is an emitter or target whose position is at $(x, y) \in \Re^{2}$ (in $\Re^{3}$ the position is $(x, y, z)$ ). And a set of measurements $z_{i}, i \in 1,2,3 \ldots N(N$ denotes the number of nodes) is obtained. The measurements can be in any form, such as distance, angle of arrival or bearing, etc from a number of sensors. In the noiseless case we have 


$$
z_{i}=f_{i}(x, y)
$$

where $f_{i}$ denotes the mapping from the target to the measurements. The function $f_{i}$ is assumed (as is reasonable) to be obtained analytically.

In practice the true value of $z_{i}$ is not available. Instead we have the noisy measurements $\tilde{z}_{i}$ defined by

$$
\tilde{z}_{i}=z_{i}+n_{i}
$$

where $n_{i}$ denotes the additive noise corrupting the measurements. This $n_{i}$ is usually assumed to be an independent ${ }^{1}$ Gaussian random variables with zero mean and known variance $\sigma_{i}^{2}$. Therefore equation (1) can be rewritten as follows.

$$
\tilde{z}_{i}=f_{i}(x, y)+n_{i}
$$

For $(x, y) \in \Re^{2}$ the equations of (3) will be overdetermined when $N \geq 3(N \geq 4$ in $3 \mathrm{D})$. In other words, there will generally be no solution to the equations of (3) except in the noiseless case. There are various methods that can be used to address this problem, such as least squares, maximum likelihood, etc [8,9].

Here we take the least squares method for example. The main idea of the method is to find the estimation of target position for which the function values best fit the data. Best is defined as when the sum of the squared error residuals is a minimum. Now the localization problem is transferred to be an optimization problem as follows.

$$
\arg \min _{x, y} \sum_{i=1}^{N} n_{i}^{2} \quad N \geq 3
$$

where

$$
n_{i}=\tilde{z}_{i}-f_{i}(x, y)
$$

If the noises have different variances, a minor variation of (4) is used, incorporating the variances as weights. By solving the above optimization equations (which is often computationally difficult) we can finally obtain the estimated position.

\section{B. High Level View of Bias}

As mentioned in the introduction, two kinds of bias can arise in localization problems. The first one is measurement bias which is caused by the environmental conditions. In this paper we assume there is no bias in the measurements. Our concern is with the second type of bias, viz. estimation bias. In this subsection, a brief view of estimation bias will be presented.

Assume $h$ is a function which maps measurements to the target position estimates. For the moment, disregard how $h$ might be determined. To explain the bias in estimation, we assume there are $M$ sets of measurements $z_{i}^{(j)}, i \in 1,2,3 \ldots N$ and $j \in 1,2,3 \ldots M$. In the absence of measurement noise and in a $2 \mathrm{D}$ situation, we have

$$
\begin{aligned}
& x_{j}=h_{1}\left(z_{1}^{(j)}, z_{2}^{(j)}, z_{3}^{(j)}, \ldots, z_{N}^{(j)}\right) \\
& y_{j}=h_{2}\left(z_{1}^{(j)}, z_{2}^{(j)}, z_{3}^{(j)}, \ldots, z_{N}^{(j)}\right)
\end{aligned}
$$

where $\left(x_{j}, y_{j}\right)$ denotes the target position corresponding to each set of measurements. In the noiseless situation $\forall j, x_{j}=$ $x, y_{j}=y$ and $z_{i}^{(j)}=z_{i}$ where $(x, y)$ is the true value of the target position and $z_{i}$ is the noiseless measurement.

\footnotetext{
${ }^{1}$ Generally, the assumption is valid. However, for some certain localization techniques such as TDOA, the noises are not independent but correlated in a standard way.
}

However, in practice, noise is inevitable. Therefore the measurement we can obtain is as follows.

$$
\tilde{z}_{i}^{(j)}=z_{i}+n_{i}^{(j)}
$$

where $\tilde{z}_{i}^{(j)}$ denotes a noisy measurement.

Rewriting the equation (5) and (6) for the noisy situation, we obtain

$$
\begin{gathered}
\tilde{x}_{j}=h_{1}\left(\tilde{z}_{1}^{(j)}, \tilde{z}_{2}^{(j)}, \tilde{z}_{3}^{(j)}, \ldots, \tilde{z}_{N}^{(j)}\right) \\
\tilde{y}_{j}=h_{2}\left(\tilde{z}_{1}^{(j)}, \tilde{z}_{2}^{(j)}, \tilde{z}_{3}^{(j)}, \ldots, \tilde{z}_{N}^{(j)}\right)
\end{gathered}
$$

where $\left(\tilde{x}_{j}, \tilde{y}_{j}\right)$ is the target position corresponding to each set of noisy measurement $\tilde{z}_{1}^{(j)}, \tilde{z}_{2}^{(j)}, \tilde{z}_{3}^{(j)}, \ldots, \tilde{z}_{N}^{(j)}$

Generally, we might average the $\tilde{x}_{j}$ and $\tilde{y}_{j}$ to obtain a position. In other words we take the expected value of $\tilde{x}_{j}$ and $\tilde{y}_{j}$ as the estimated target position. Here we take $\tilde{x}_{j}$ for example:

$$
E[\tilde{x}]=E\left[h_{1}\left(\tilde{z}_{1}, \tilde{z}_{2}, \tilde{z}_{3}, \ldots, \tilde{z}_{N}\right)\right]
$$

However, if the function $h_{1}$ is nonlinear we obtain

$$
\begin{aligned}
E[\tilde{x}] & =E\left[h_{1}\left(\tilde{z}_{1}, \tilde{z}_{2}, \tilde{z}_{3}, \ldots, \tilde{z}_{N}\right)\right] \\
& \neq h_{1}\left(E\left[\tilde{z}_{1}, \tilde{z}_{2}, \tilde{z}_{3}, \ldots, \tilde{z}_{N}\right]\right) \\
& =h_{1}\left(z_{1}, z_{2}, z_{3}, \ldots, z_{N}\right) \\
& =x
\end{aligned}
$$

Similarly we have $E[\tilde{y}] \neq y$. Therefore the bias appears in the estimate.

$$
\text { Bias }_{x}=E[\tilde{x}]-x \quad \text { Bias }_{y}=E[\tilde{y}]-y
$$

This bias can be used to systematically correct any single estimate from any single set of measurements. From the above analysis, we can see there are two main factors causing the estimation bias. The first one is the noise in measurements. The other factor is that the functions $h_{1}$ and $h_{2}$ are usually nonlinear. This leads to the inequality.

In this subsection a brief high level view of bias is presented. How to formulate the bias analytically and how to correct the bias in estimates will be discussed in the next section.

\section{Determining Bias in Localization Algorithms}

The bias-correction method will be proposed in this section. The analysis will be done in two situations based on rangemeasurement localization algorithms for illustration purposes. The first situation with one target and two sensors whose locations have already been specified will be presented in subsection III.A. Then in III.B the situation with three anchors will be described in details. Further, we extend our method to the situation with more than three anchors in subsection III.C. For ease of exposition in presenting the method, we restrict attention to Cartesian coordinates.

\section{A. Two Anchors Situation}

A scenario with two range-measuring anchors and one target is considered in this subsection. The noisy range measurements from two anchors define two circles with two points of intersection. Only one of them is the target estimate. Here we assume further information has been obtained such as an a priori area restriction to resolve this ambiguity problem.

Assume $f_{1}, f_{2}$ (which together form a vector function $f$ ) are the mappings from target to measurement distance. We can obtain the following equations according to the simple geometric relationships.

$$
d_{i}=f_{i}(x, y)=\sqrt{\left(x-x_{i}\right)^{2}+\left(y-y_{i}\right)^{2}} \quad i=1,2
$$

where $x, y$ denote the position of target, while $x_{i}, y_{i}$ denotes the known position of anchor $i$. Further $d_{i}$ (which correspond 
to $z_{i}$ in Section II) is the Euclidean distances between target and anchor $i$.

Suppose the inverse functions of $f_{1}, f_{2}$ are $g_{1}, g_{2}$. It is reasonable to regard the vector function $g$ as in fact a localization mapping, i.e. a function delivering a position estimate from the measurements. Thus $g$ is a particular form of the function $h$ mentioned in Section II. Here we have:

$$
\begin{aligned}
& x=g_{1}\left(d_{1}, d_{2}\right) \\
& y=g_{2}\left(d_{1}, d_{2}\right)
\end{aligned}
$$

It can be verified that there are derivatives of any order of $g_{1}$ and $g_{2}$.

The true values of $d_{1}$ and $d_{2}$ are not available in practice. Only the noisy measurements can be obtained:

$$
\tilde{d}_{i}=d_{i}+n_{i} \quad i=1,2
$$

where $n_{i}$ is the measurement error which is an independent Gaussian random variable with zero mean and variance $\sigma_{d_{i}}^{2}$.

Now we can expand $g_{1}$ and $g_{2}$ about the point $\left(d_{1}, d_{2}\right)$ by Taylor series. Suppose the Taylor series is truncated to second order in the $n_{i}$. Here we take $x$ as example.

$$
\begin{aligned}
x+\Delta x= & g_{1}\left(\tilde{d}_{1}, \tilde{d}_{2}\right) \\
= & g_{1}\left(d_{1}+n_{1}, d_{2}+n_{2}\right) \\
= & g_{1}\left(d_{1}, d_{2}\right)+\left[\frac{\partial g_{1}}{\partial d_{1}} n_{1}+\frac{\partial g_{1}}{\partial d_{2}} n_{2}\right] \\
& +\frac{1}{2 !}\left[\left(n_{1}\right)^{2} \frac{\partial^{2} g_{1}}{\partial d_{1}^{2}}+2 n_{1} n_{2} \frac{\partial^{2} g_{1}}{\partial d_{1} d_{2}}+\left(n_{2}\right)^{2} \frac{\partial^{2} g_{1}}{\partial d_{2}^{2}}\right]
\end{aligned}
$$

From the above equations we can obtain an approximation for the expected value of $\Delta x$ as follows.

$$
E(\Delta x)=\frac{1}{2 !}\left[\left(\sigma_{d_{1}}\right)^{2} \frac{\partial^{2} g_{1}}{\partial d_{1}^{2}}+\left(\sigma_{d_{2}}\right)^{2} \frac{\partial^{2} g_{1}}{\partial d_{2}^{2}}\right]
$$

$E(\Delta y)$ can be obtained in the same way. Here $E(\Delta x)$ and $E(\Delta y)$ are considered as the bias. The tensor form of the bias can be obtained in [7].

For range-measurement localization in a $2 \mathrm{D}$ situation, to compute the derivatives of the localization mappings ( $g_{1}$ and $g_{2}$ ) analytically is not very difficult. However, when one considers for example a three dimensional problem involving TDOA and bearing data, the calculation of the inverse mappings and their derivatives would be much harder. In fact, in almost all cases, it is much easier to obtain the derivatives of forward mappings $\left(f_{1}\right.$ and $\left.f_{2}\right)$. Therefore we consider how use $f_{1}, f_{2}$ and their derivatives to compute the derivatives of $g_{1}$ and $g_{2}$. To achieve this, we need to use a Jacobian matrix.

The following equations derive from one property of the Jacobian matrix.

$$
\left[\begin{array}{ll}
\frac{\partial f_{1}}{\partial x} & \frac{\partial f_{1}}{\partial y} \\
\frac{\partial f_{2}}{\partial x} & \frac{\partial f_{2}}{\partial y}
\end{array}\right]\left[\begin{array}{ll}
\frac{\partial g_{1}}{\partial d_{1}} & \frac{\partial g_{1}}{\partial d_{2}} \\
\frac{\partial g_{2}}{\partial d_{1}} & \frac{\partial g_{2}}{\partial d_{2}}
\end{array}\right]=\left[\begin{array}{ll}
1 & 0 \\
0 & 1
\end{array}\right]
$$

In order to simplify the equations we use $A, B, C, D$ and $a, b, c, d$ to denote the derivatives of $f_{1}, f_{2}, g_{1}$ and $g_{2}$ respectively.

$$
\begin{gathered}
A=\frac{\partial g_{1}}{\partial d_{1}}, B=\frac{\partial g_{1}}{\partial d_{2}}, C=\frac{\partial g_{2}}{\partial d_{1}}, D=\frac{\partial g_{2}}{\partial d_{2}} \\
a=\frac{\partial f_{1}}{\partial x}, b=\frac{\partial f_{1}}{\partial y}, c=\frac{\partial f_{2}}{\partial x}, d=\frac{\partial f_{2}}{\partial y}
\end{gathered}
$$

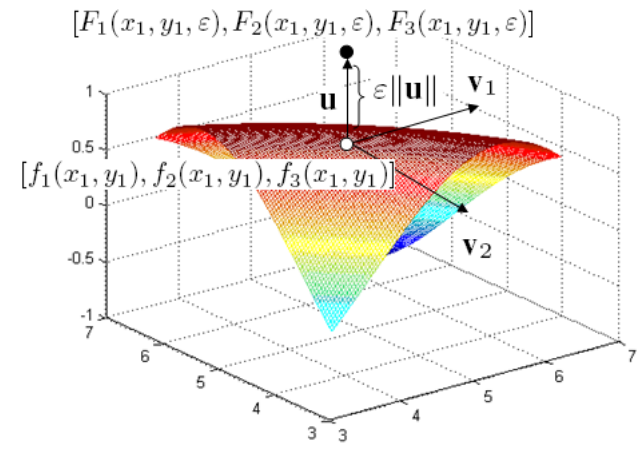

Fig. 1. Introduce One Variable

By solving the above equations we can obtain:

$$
\frac{\partial^{2} g_{1}}{\partial d_{1}^{2}}=\frac{A_{x} d-A_{y} c}{a d-b c}
$$

where $A_{x}$ denotes $\frac{\partial A}{\partial x}, A_{y}$ denotes $\frac{\partial A}{\partial y}$ and $A=\frac{\partial g_{1}}{\partial d_{1}}=\frac{d}{a d-b c}$.

Other derivatives of $g_{1}$ and $g_{2}$ have a similar form. Substituting the formulas for the derivatives of $g_{1}, g_{2}$ computed by the derivatives of $f_{1}$ and $f_{2}$ into $E(\Delta x)$ and $E(\Delta y)$, we can finally obtain the equations of bias including derivatives of $f_{1}$ and $f_{2}$ which are much easier to compute.

The accuracy of the bias calculation requires that the Taylor series truncation does not cause significant error. The $j$-th order terms in the Taylor series can be expressed using derivatives of $f$, multiplied by the $\mathrm{j}$-th power of the inverse of the Jacobian determinant of $f$. When this determinant is small, there is potentially more approximation error. A convenient measure is provided by the mean square error (MSE) matrix.

$$
E\left(\left[\begin{array}{c}
\Delta x \\
\Delta y
\end{array}\right]\left[\begin{array}{ll}
\Delta x & \Delta y
\end{array}\right]\right)=J^{-1}\left[\begin{array}{cc}
\sigma_{d_{1}}^{2} & 0 \\
0 & \sigma_{d_{2}}^{2}
\end{array}\right]\left(J^{-1}\right)^{T}
$$

where

$$
J=\left[\begin{array}{ll}
\frac{\partial f_{1}}{\partial x} & \frac{\partial f_{1}}{\partial y} \\
\frac{\partial f_{2}}{\partial x} & \frac{\partial f_{2}}{\partial y}
\end{array}\right]
$$

which is the sum of the bias vector times its transpose and the covariance of the position error. When the first term of the sum is small in relation to the sum itself, then the bias calculation may not be very accurate. In the simulations, we observe that at a certain empirically determined threshold for the determinant of the MSE, the bias calculation does become inaccurate.

\section{B. Three Anchors Situation}

Suppose now there are three range-measuring anchors and one target. In the noiseless case, the three circles defined by the range measurements will generically have a single point of intersection (an exception occurs if the three anchors are collinear). In the noisy case (to be assumed here), generally, a common point of intersection of the three circles does not exist. Furthermore, the equations are overdetermined which means there are more scalar measurements than there are unknowns. We no longer have an inverse mapping and thus cannot use the Jacobian matrix. The localization mapping is determined by solving a minimization problem and is difficult or even impossible to formulate analytically.

In order to resolve these problems, we need to introduce an extra variable into the mapping set. 
Assume a surface (shown in Fig. 1) consists of points which corresponding to sets of noiseless measurements $\left(d_{1}, d_{2}, d_{3}\right)$, where

$$
d_{i}=f_{i}(x, y) \quad i=1,2,3
$$

However, in the noisy case, errors exist in the measurements. Therefore we have

$$
\tilde{d}_{i}=d_{i}+n_{i}=f_{i}(x, y)+n_{i} \quad i=1,2,3
$$

where $\tilde{d}_{i}$ is the noisy measurement while $n_{i}$ denotes the error in measurements. The error is an independent Gaussian variable with zero mean and variance $\sigma_{d_{i}}^{2}$.

According to the least squares method mentioned in II.B, to resolve this overdetermined equations we need to solve an optimization problem, determine

$$
\arg \min _{x, y} \sum_{i=1}^{3} n_{i}^{2}
$$

where

$$
n_{i}=\tilde{d}_{i}-f_{i}(x, y) \quad i=1,2,3
$$

The least squares method, in fact, attempts to find a point $\left(f_{1}(x, y), f_{2}(x, y), f_{3}(x, y)\right)$ on the surface corresponding to a set of noisy measurement $\left(\tilde{d}_{1}, \tilde{d}_{2}, \tilde{d}_{3}\right)$ (off the surface) to minimize the distance between the two points.

Assume, in the Fig. 1, the black point denotes a set of noisy measurements, and the white point is the corresponding point on the surface ${ }^{2}$. The black point must be on the normal vector to the surface passing through the white one. The distance between the two points can be denoted as $\varepsilon\|\mathbf{u}\|=$ $\sqrt{n_{1}^{2}+n_{2}^{2}+n_{3}^{2}}$, where $\mathbf{u}$ is a normal vector at the white point and $\varepsilon$ is a coefficient to minimize the distance. The normal vector $\mathbf{u}$ can be calculated as follows.

At the white point we can obtain two tangent vectors $\mathbf{v}_{1}$ and $\mathbf{v}_{2}$ as follows.

$$
\mathbf{v}_{1}=\left(\begin{array}{c}
\frac{\partial f_{1}}{\partial x} \\
\frac{\partial f_{2}}{\partial x} \\
\frac{\partial f_{3}}{\partial x}
\end{array}\right), \mathbf{v}_{2}=\left(\begin{array}{c}
\frac{\partial f_{1}}{\partial y} \\
\frac{\partial f_{2}}{\partial y} \\
\frac{\partial f_{3}}{\partial y}
\end{array}\right)
$$

By cross multiplying the two vectors, we can obtain the normal vector $\mathbf{u}$.

$$
\mathbf{u}=\mathbf{v}_{1} \times \mathbf{v}_{2}=\left(\begin{array}{l}
\frac{\partial f_{2}}{\partial x} \frac{\partial f_{3}}{\partial y}-\frac{\partial f_{3}}{\partial x} \frac{\partial f_{2}}{\partial y} \\
\frac{\partial f_{3}}{\partial x} \frac{\partial f_{1}}{\partial y}-\frac{\partial f_{1}}{\partial x} \frac{\partial f_{3}}{\partial y} \\
\frac{\partial f_{1}}{\partial x} \frac{\partial f_{2}}{\partial y}-\frac{\partial f_{2}}{\partial x} \frac{\partial f_{1}}{\partial y}
\end{array}\right)
$$

Note that $f_{1}(x, y), f_{2}(x, y)$ and $f_{3}(x, y)$ can be readily written down according to simple geometric relationships. Therefore a new set of functions $F_{1}, F_{2}, F_{3}$ for noisy measurements can be obtained through moving the $f_{1}, f_{2}$ and $f_{3}$ along the normal vector for a minimal distance $\varepsilon\|\mathbf{u}\|$. The new set of functions determine equations which are no longer overdetermined because an extra variable $\varepsilon$ has been introduced.

Now we have the the forward mapping $F: R^{3} \rightarrow R^{3}$ and its inverse $G: R^{3} \rightarrow R^{3}$ as follows.

$$
d_{1}=F_{1}(x, y, \varepsilon)=f_{1}(x, y)+\varepsilon\left(\frac{\partial f_{2}}{\partial x} \frac{\partial f_{3}}{\partial y}-\frac{\partial f_{3}}{\partial x} \frac{\partial f_{2}}{\partial y}\right)
$$

\footnotetext{
${ }^{2}$ Sometimes the corresponding point is not unique. At that time we assume further information can be obtained to resolve this ambiguity.
}

$$
\begin{aligned}
d_{2}=F_{2}(x, y, \varepsilon)= & f_{2}(x, y)+\varepsilon\left(\frac{\partial f_{3}}{\partial x} \frac{\partial f_{1}}{\partial y}-\frac{\partial f_{1}}{\partial x} \frac{\partial f_{3}}{\partial y}\right) \\
d_{3}=F_{3}(x, y, \varepsilon)= & f_{3}(x, y)+\varepsilon\left(\frac{\partial f_{1}}{\partial x} \frac{\partial f_{2}}{\partial y}-\frac{\partial f_{2}}{\partial x} \frac{\partial f_{1}}{\partial y}\right) \\
x & =G_{1}\left(d_{1}, d_{2}, d_{3}\right) \\
y & =G_{2}\left(d_{1}, d_{2}, d_{3}\right) \\
\varepsilon & =G_{3}\left(d_{1}, d_{2}, d_{3}\right)
\end{aligned}
$$

Note that while $F$ can be analytically computed this is almost certainly difficult or impossible for $G$. We can obtain the bias formula in the same way as in the two anchors situation by using a Taylor series. Here we take $E(\Delta x)$ for example ( $\Delta y$ can be obtained in the same way):

$$
E(\Delta x)=\frac{1}{2 !}\left(\sigma_{d_{1}} \frac{\partial}{\partial d_{1}}+\sigma_{d_{2}} \frac{\partial}{\partial d_{2}}+\sigma_{d_{3}} \frac{\partial}{\partial d_{3}}\right)^{2} G_{1}\left(d_{1}, d_{2}, d_{3}\right)
$$

The processes to follow are similar to the two anchors situation. In particular we can calculate the derivatives of $G_{1}$, $G_{2}$ and $G_{3}$ using $F_{1}, F_{2}, F_{3}$ and their derivatives. The MSE matrix is also selected as the performance criterion in the three anchors situation.

\section{More than Three Anchors}

When there are more than three anchors, the situation is similar to the three anchors case except that the extra variable $\varepsilon$ is no longer a scalar. Instead it is a vector which can be defined as follows.

$$
\varepsilon=\left[e_{1}, e_{2}, \ldots, e_{i}\right]^{T} \quad i=m-2
$$

where $e_{i}$ denotes a coefficient to minimize the moved distance in each dimension of the normal, and $m$ denotes the number of anchors.

The other processes are as same as got the three anchors situation. We omit the details here.

\section{Simulation Results}

In this section the results of Monte Carlo simulations will be provided corresponding to the two situations in Section II. For each situation we have done two types of simulation. In the first one we vary the $x$ value of the target while fixing the $y$-position. In the second simulation the $x$ value of the target is fixed while the $y$ value is adjusted. The results verify the proposed method can correct the bias very well.

\section{A. Simulation Set-up}

Assumption

- The measurement errors for $d_{1}$ and $d_{2}$ (in three anchors situation there will be an extra $d_{3}$ ) are produced by independent Gaussian distributions $\left(\mu=0\right.$ and $\left.\sigma^{2}=1^{3}\right)$. If the absolute value of the error is larger than $5 \%$ of the measurement distance, the measurement is rejected. In other words, there is a upper threshold for errors.

Definition:

- Expected target position: In the simulation we have thousands of distance measurements: $d_{1 i}(i=1,2,3 \ldots N)$ and $d_{2 i}(i=1,2,3 \ldots N)$. By averaging them we can obtain the mean value of $d_{1}$ and $d_{2}: \bar{d}_{1}=\frac{1}{N} \sum d_{1 i}(i=1,2,3 \ldots N)$ and $\bar{d}_{2}=\frac{1}{N} \sum d_{2 i}(i=1,2,3 \ldots N)$. Now we can obtain $x=f_{1}\left(\bar{d}_{1}\right)$ and $y=f_{2}\left(\bar{d}_{2}\right)$. Here $(x, y)$ is termed the

\footnotetext{
${ }^{3}$ The effect made by adjusting the levels of $\sigma^{2}$ will be studied in our future
} work 


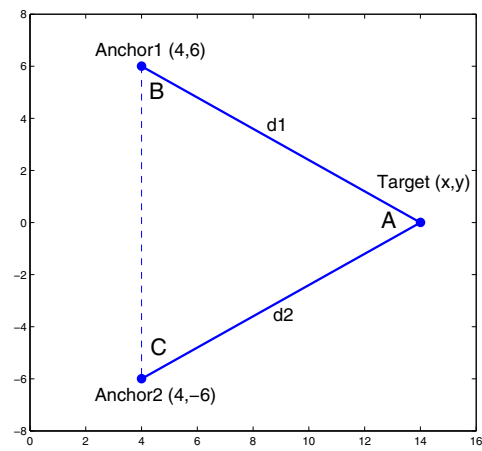

Fig. 2. Geometry of the anchors and the target

expected target position, and when $N \rightarrow \infty$, the expected target position becomes the true position.

- Estimated target position: In the simulation we have thousands of distance measurements: $d_{1 i}(i=1,2,3 \ldots N)$ and $d_{2 i}(i=1,2,3 \ldots N)$. For each of them we have: $x_{i}=f_{1}\left(d_{1 i}\right)(i=1,2,3 \ldots N)$ and $y_{i}=f_{2}\left(d_{2 i}\right)(i=$ $1,2,3 \ldots N)$. To average $x_{i}$ and $y_{i}$, we can obtain the mean value: $\bar{x}=\frac{1}{N} \sum x_{i}(i=1,2,3 \ldots N)$ and $\bar{y}=\frac{1}{N} \sum y_{i}(i=$ $1,2,3 \ldots N)$. The position $(\bar{x}, \bar{y})$ is termed the estimated target position.

- Bias-corrected target position: For each $x_{i}=f_{1}\left(d_{1 i}\right)(i=$ $1,2,3 \ldots N)$ and $y_{i}=f_{2}\left(d_{2 i}\right)(i=1,2,3 \ldots N)$, we use the proposed method to correct the bias. Therefore we can obtain $x_{i}^{c}=x_{i}-x_{i}^{\text {bias }}(i=1,2,3 \ldots N)$ and $y_{i}^{c}=$ $y_{i}-y_{i}^{\text {bias }}(i=1,2,3 \ldots N)$. The mean value of $x_{i}^{c}(i=$ $1,2,3 \ldots N)$ and $y_{i}^{c}(i=1,2,3 \ldots N)$ is the bias-corrected target position.

- Analytical Bias: The $x_{i}^{b i a s}(i=1,2,3 \ldots N)$ and $y_{i}^{\text {bias }}(i=$ $1,2,3 \ldots N)$, which is calculated through the analytical formulation of bias, is termed the analytical bias.

- Experimental Bias: The distance between the expected target position and the estimated one.

- $\hat{d}$ : The absolute distance between the estimated target position and the expected one.

- $d_{c}$ : The absolute distance between the bias-corrected target position and the expected one.

\section{B. Two Anchors Situation}

Fig. 2 shows the geometry of the two anchors and the target. We fix the two anchors at $(4,6)$ and $(4,-6)$ in both cases.

1. First Simulation

In this simulation we fix the value of $y$ of the target at zero while changing the value of $x$, i.e. we adjust the angle A. The variation of angle $\mathrm{A}$ is from $20^{\circ}$ to $170^{\circ}$. Following are the simulation results.

Fig. 3 illustrates the comparison of $\hat{d}$ and $d_{c}$. In the figure $d_{c}$ is smaller than $\hat{d}$ all the time (from $20^{\circ}$ to $140^{\circ}$ ) which means the proposed method can correct the bias very well. However, when the angle $\mathrm{A}$ is very large (larger than $140^{\circ}$ ) our approach cannot work (see TABLE I). At that time the target is too close to the link line of the two anchors so that the target and the two anchors can be considered as nearly collinear. Localization algorithms often are unsatisfactory in this circumstance [10].

Fig. 4 shows the comparison of experimental bias and analytical bias. From $20^{\circ}$ to $140^{\circ}$, the experimental bias is almost equal to the analytical one. This also verifies, from another standpoint, the proposed method is effective.

Fig. 5 depicts the determinant of the MSE (mean square error) matrix. The determinant of the MSE is smaller than the threshold (here 3.5) except when the angle $\mathrm{A}$ is $20^{\circ}$. When

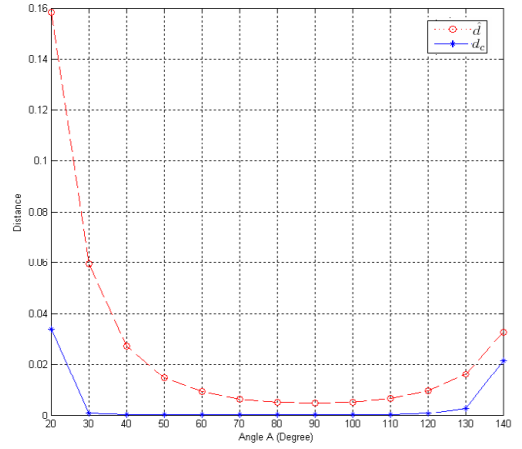

Fig. 3. Comparison of $\hat{d}$ and $d_{c}$

\begin{tabular}{|c|c|c|c|}
\hline Angle A (Degree) & $150^{\circ}$ & $160^{\circ}$ & $170^{\circ}$ \\
\hline$\hat{d}$ & 0.0676 & 0.0789 & 0.0830 \\
\hline$d_{c}$ & 9.3072 & 20.7972 & 162.6368 \\
\hline
\end{tabular}

TABLE I

COMPARISON OF $\hat{d}$ AND $d_{c}$

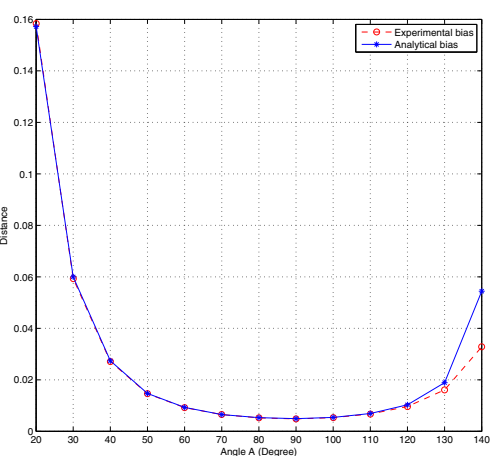

Fig. 4. Comparison of Experimental bias and Analytical bias

the angle $\mathrm{A}$ is smaller than $20^{\circ}$, the determinant of the MSE matrix will be larger than the threshold (see TABLE II), which means the result is inaccurate even if $d_{c}$ is smaller than $\hat{d}$ (e.g. when $\mathrm{A}=20^{\circ}$ ). At that time, the target is in a far field compared to the two anchors, and again the anchors and the target are almost collinear, where localization algorithms are often ineffective.

\section{Second Simulation}

In the second simulation we set the value of $x$ of the target as $6 \sqrt{3}+4$ while adjusting the value of $y$, which means the angle $\mathrm{B}$ is changing: the variation of the angle $\mathrm{B}$ is between $60^{\circ}$ and $160^{\circ}$

Fig. 6 shows the comparison of $\hat{d}$ and $d_{c}$. From the figure we can conclude that the proposed method can correct the bias from $60^{\circ}$ to $130^{\circ}$. However, when angle $\mathrm{B}$ becomes larger than $130^{\circ}$ the proposed method is less effective or ineffective (see TABLE III). This is because at that time, the target is in a far field compared to the two anchors, which results in near-collinear situation.

Fig. 7 illustrates the comparison of experimental bias and analytical bias. In the figure the experimental bias is almost equal to the analytical one for most positions of B. Similarly to the first simulation, this comparison verifies the effectiveness of our approach from another point of view.

Note that from $60^{\circ}$ to $130^{\circ}$, the determinant of the MSE is smaller than the upper threshold (here 3.5).

To sum up, from the above two simulations we observe that the proposed method can correct the bias in most situations except near-collinear one. At that time, localization algorithms 


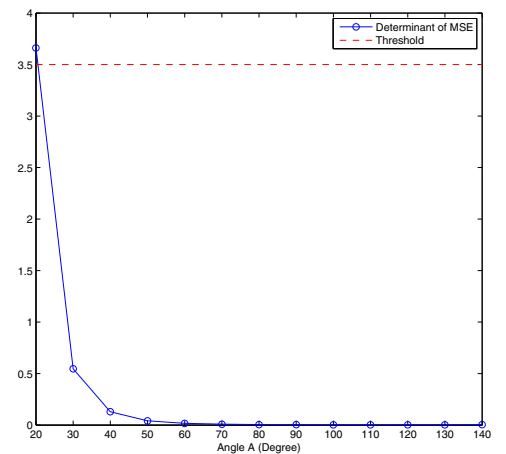

Fig. 5. Determinant of MSE (mean square error) matrix

\begin{tabular}{|c|c|c|c|}
\hline Angle A (Degree) & $10^{\circ}$ & $15^{\circ}$ & $20^{\circ}$ \\
\hline Determinant of MSE & 31.7045 & 11.0982 & 3.6553 \\
\hline
\end{tabular}

TABLE II

DETERMINANT OF MSE

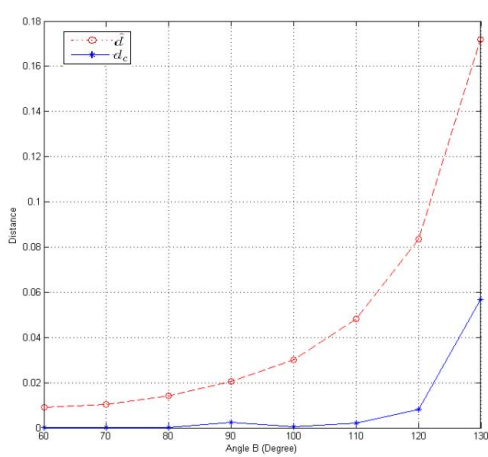

Fig. 6. Comparison of $\hat{d}$ and $d_{c}$

often can not work. In other words the bias-correction method is consistent with the applicability of localization algorithms.

\section{Three Anchors Situation}

In this situation, we fix the three anchors at $(0,-6),(0,6)$ and $(10,0)$. We also set the upper threshold of the measurement error as $3 \%$. The geometry of the anchors and the target is shown in Fig. 8.

1. First Simulation

In this simulation, similarly to the two anchors situation, we fix the value of $y$ of the target at zero while adjusting the value of $x$ in order to investigate the influence of $x$ changing. The variation of $x$ is from -3 to 28 .

Fig. 9 shows the comparison of $\hat{d}$ and $d_{c}$, with $d_{c}$ always smaller than $\hat{d}$. The proposed method can correct the bias very well. However when the absolute value of $x$ is very large (larger than 58), the determinant of the MSE matrix will be larger than the threshold (see TABLE IV), which means the result is inaccurate even if $d_{c}$ is smaller than $\hat{d}$ (e.g. when $x=58, d_{c}=0.1186$ and $\left.\hat{d}=0.3014\right)$. At that time the target is far away from the three anchors. Localization algorithms do not work satisfactorily because the target and the three anchors can be considered as nearly collinear.

Fig. 10 illustrates the comparison of experimental bias and analytical bias. From -3 to 28 , the experimental bias is very close to the analytical one. This also verifies, from another standpoint, that the proposed method is effective. The determinant of MSE matrix in this situation is always close to zero throughout the interval.

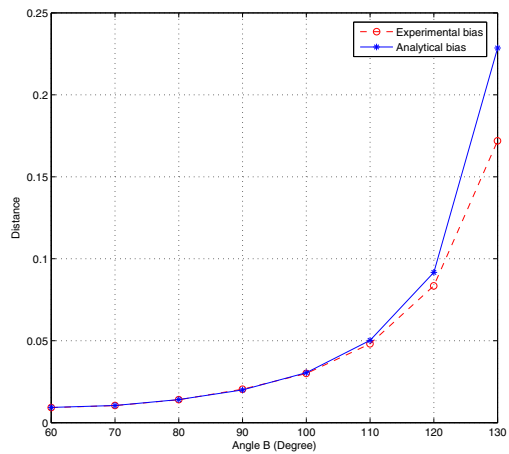

Fig. 7. Comparison of Experimental bias and Analytical bias

\begin{tabular}{|c|c|c|c|}
\hline Angle B (Degree) & $140^{\circ}$ & $150^{\circ}$ & $160^{\circ}$ \\
\hline$\hat{d}$ & 0.4212 & 0.7148 & 1.1557 \\
\hline$d_{c}$ & 8.8690 & 54.4756 & 72.7876 \\
\hline
\end{tabular}

TABLE III

COMPARISON OF $\hat{d}$ AND $d_{c}$

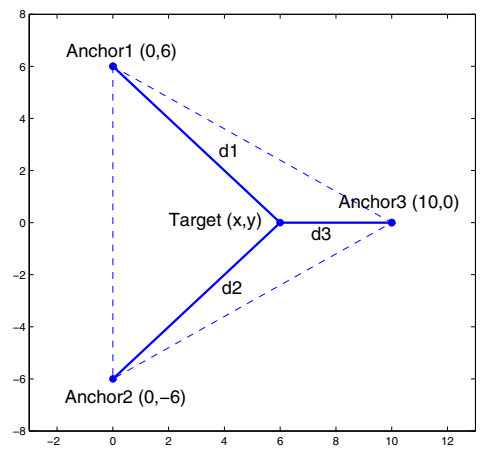

Fig. 8. First Simulation

\section{Second Simulation}

In the second simulation, we fix the value of $x$ of the target at 6 while adjusting the value of $y$ in order to investigate the influence made by $y$ changing. In light of the symmetry we set the variation of $y$ from 0 to 14 .

Fig. 11 depicts the comparison of $\hat{d}$ and $d_{c}$. From the figure we can obtain that the method can correct the bias when the value of $y$ changes from 0 to 14 . However when the absolute value of $y$ is too large (larger than 25), the proposed method is no longer effective (see TABLE V). Again at that time, the three anchors and the target can be considered as nearly collinear.

Fig. 12 shows the comparison of experimental bias and analytical bias. The experimental bias is close to the analytical one when the $y$ value of the target changes from 0 to 14 . From another standpoint, the proposed method is verified to be effective. The determinant of MSE matrix is also close to zero from 0 to 14 .

From the above two simulations we can draw a conclusion that, similarly to the two anchors situations the performance of the proposed method is also consistent with localization algorithms in the three anchors situation. In other words the proposed method can correct the bias very well in most situations except for the nearly collinear one. Compared to the two anchors situation, the determinant of the MSE matrix is much smaller in corresponding cases. Therefore, the results in the three anchors situation are more accurate. 


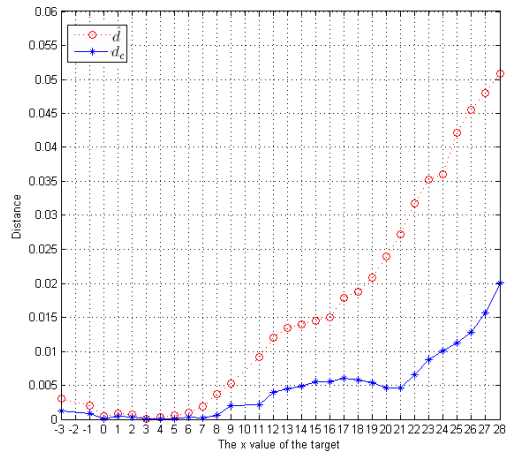

Fig. 9. Comparison of $\hat{d}$ and $d_{c}$

\begin{tabular}{|c|c|c|c|c|}
\hline$x$ value of the target & -60 & -58 & 58 & 60 \\
\hline The Determinant of MSE & 3.9689 & 3.5953 & 3.8962 & 4.0465 \\
\hline
\end{tabular}

TABLE IV

The Determinant of MSE Matrix

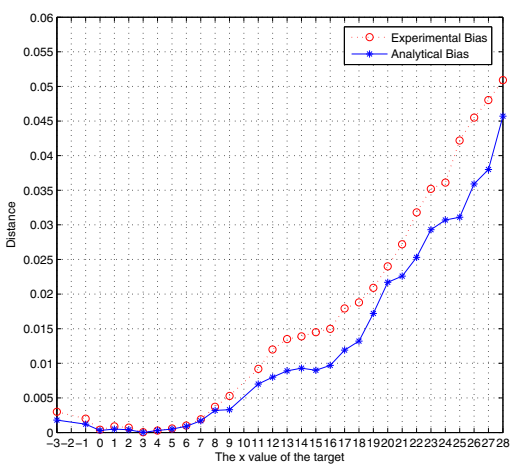

Fig. 10. Comparison of Experimental bias and Analytical bias

\section{Conclusions}

This paper proposed a method to correct the bias in localization algorithms, where an analytical formula for the localization mapping from measurements to estimated target position may not be available. We formulated the bias in a simple way through a Taylor series and Jacobian matrix. The proposed method is analyzed in two situations. In the first situation there are two anchors and one target. Further information is assumed to be obtained in order to resolve the ambiguity. When there are three anchors, the relevant equation set is overdetermined, and if the measurements are noisy there will be no solution to the equation set. To resolve this problem, we introduce an extra variable into the equations corresponding to adopting a least squares approach. The Monte Carlo simulations verify the proposed method can correct the bias in most situations except for the nearly collinear one. At that time localization algorithms themselves often cannot work. In other words, the proposed method is consistent with localization algorithms. Though the proposed method is analyzed in distance-based localization algorithms, it can be extended to other localization algorithms. In other words, the proposed method can serve as an add-on for most of the existing localization algorithms. Our future work is to analyze the performance of the proposed method in different coordinate systems. Furthermore, we will consider truncation of the Taylor series to higher orders in order to examine the effect of different levels of noise variance.

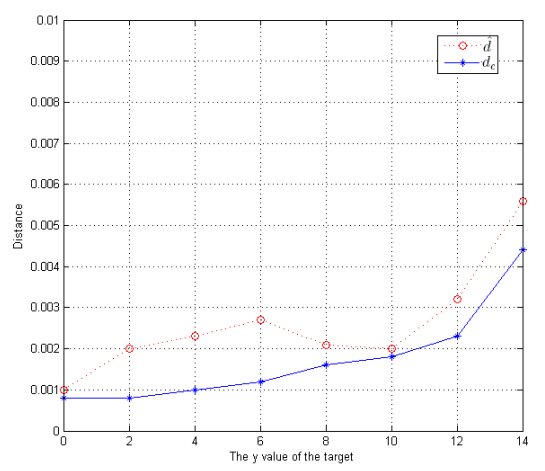

Fig. 11. Comparison of $\hat{d}$ and $d_{c}$

\begin{tabular}{|c|c|c|c|}
\hline$y$ value of the target & 25 & 30 & 35 \\
\hline$\hat{d}$ & 0.0141 & 0.0138 & 0.0117 \\
\hline$d_{c}$ & 0.0181 & 0.0385 & 0.0554 \\
\hline
\end{tabular}

TABLE V

COMPARISON OF $\hat{d}$ AND $d_{c}$

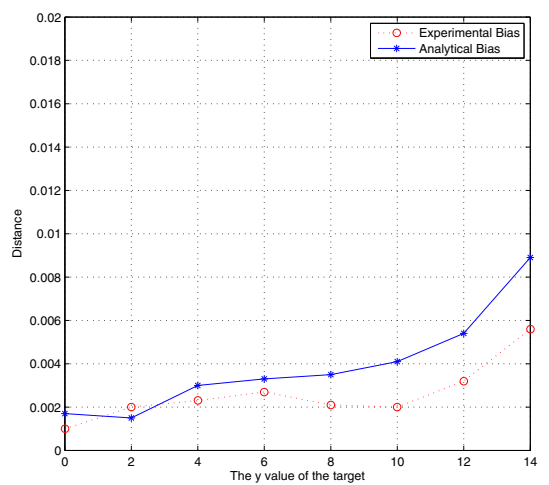

Fig. 12. Comparison of Experimental bias and Analytical bias REFERENCES

[1] G. Mao, B. Fidan and Brian D.O. Anderson. Wireless sensor network localization techniques. Computer Networks, 51:2529-2553, 2007.

[2] K. Doğançay. Online Optimization of Receiver Trajectories for ScanBased Emitter Localization. IEEE Transactions on Aerospace and Electronic Systems, 43(3): 1117-1125, 2007.

[3] J. S. Picard and A. J. Weiss. Localization of networks using various ranging bias models. Wireless Communications and Mobile Computing, 8: 553-562, 2008.

[4] X. Lin, Y. Bar-Shalom and T. Kirubarajan. Multisensor-Multitarget Bias Estimation for General Asynchronous Sensors. IEEE Transactions on Aerospace and Electronic Systems, 41(3): 899-921, 2005.

[5] M. Gavish and A.J. Weiss. Performance analysis of bearing-only target location algorithms. IEEE Transactions on Aerospace and Electronic Systems, 28(3): 817-827, 1992.

[6] K. Doğançay and S. P. Drake. Bias reduction for the scan-based leastsquares emitter localization algorithm. Proc. 14th European Signal Processing Conference, EUSIPCO 2006, 5 pp., 2006.

[7] S. P. Drake and K. Doğançay. Some Applications of Tensor Algebra to Estimation Theory. 3rd International Symposium on Wireless Pervasive Computing, ISWPC 2008, 106-110, 2008.

[8] W. H. Foy. Position-Location Solution by Taylor-Series Estimation. IEEE Transactions on Aerospace and Electronic Systems, AES-12(2): 187-194, 1976.

[9] D. J. Torrieri. Statistical Theory of Passive Location Systems. IEEE Transactions on Aerospace and Electronic Systems, AES-20(2): 183-198, 1984.

[10] B. Fidan, S. P. Drake, G. Mao, B. D. O. Anderson and A. A Kannan. Collinearity problems in passive target localization using direction finding sensors. Submitted to IEEE Symposium on Computational Intelligence for Security and Defense Applications, Ottawa, Canada, July 2009. 